\title{
DETECCIÓN DE VEHÍCULOS BASADA EN VISIÓN POR COMPUTADOR PARA SISTEMA DE AYUDA A LA CONDUCCIÓN EN TRÁFICO URBANO. GENERACIÓN DE HIPÓTESIS.
}

\author{
M. Ibarra-Arenado ${ }^{1}$, T. Tjahjadi ${ }^{2}$, S. Robla-Gómez ${ }^{1}$, J.M. Pérez-Oria ${ }^{1}$ \\ ${ }^{1}$ Departamento de Tecnología Electrónica e Ingeniería de Sistemas y Automática, Universidad de Cantabria, \\ Santander, Cantabria, Avda. los Castros s/n, 39005, SPAIN \\ ${ }^{2}$ School of Enginering, University of Warwick, Coventry CV4 7AL, UNITED KINGDOM \\ E-mail: [ibarra,srobla,oria]@teisa.unican.es, t.tjahjadi@warwick.ac.uk
}

\begin{abstract}
Resumen
La detección de vehículos es una tarea fundamental en los sistemas anticolisión frontal. Los métodos de detección de vehículos basados en visión por computador se dividen generalmente en dos etapas: generación de hipótesis y verificación de hipótesis. El presente artículo se centra en la primera, presentando un método de generación de hipótesis basado en la sombra que los vehículos originan bajo sí mismos. La detección de la sombra se realiza mediante un nuevo enfoque de umbralización por intensidad. En primer lugar, un umbral es establecido a partir de la región de la carretera frente al ego-vehículo. Posteriormente, un segundo umbral es empleado sobre cada candidato con el fin de identificar la posible sombra lateral adyacente al vehículo. El método supera importantes dificultades como la presencia de sombras laterales, indicaciones pintadas en el asfalto $y$ fondos de imagen saturados de objetos. Ensayos realizados en tráfico urbano muestran un comportamiento eficiente y fiable del método.
\end{abstract}

Palabras Clave: Visión por computador, detección de vehículos, sistemas de ayuda a la conducción.

\section{INTRODUCCIÓN}

Con el fin de evitar colisiones por alcance, los sistemas anticolisión frontal (FCAS) ayudan al conductor a mantener la distancia de seguridad con el vehículo delantero que circula en la trayectoria. La detección de vehículos basada en visión por computador para sistemas FCAS es en la actualidad un gran desafío. Aunque muchos métodos han sido propuestos, todavía supone un campo de investigación activo, donde los esfuerzos se dirigen a lograr sistemas más fiables y rápidos.

Son diversos los factores que dificultan la detección de vehículos en una imagen. Por un lado, el tamaño, la forma y el color de los mismos varía en función de la marca y modelo, por lo que la detección no se puede reducir a la búsqueda de un vehículo con unas características únicas, sino que se ha de ampliar a características comunes a todos los modelos. Por otro lado, el ego-vehículo (vehículo con el sistema instalado) está en movimiento, por lo que el escenario además de ser desconocido, está constantemente cambiando. Esto impide el uso de técnicas como la sustracción del fondo de imagen, la cual es habitual en aplicaciones con cámara fija. Finalmente, los sistemas FCAS operan en exteriores estando muy condicionados por la iluminación, la cual es desconocida (día soleado, nublado, etc) e incontrolable. Esta puede originar sombras y brillos en la carretera y en los objetos de la escena, lo que en la imagen puede ocasionar la unión aparente entre los mismos, así como alterar sus características de intensidad, cromaticidad e incluso forma.

Generalmente, los métodos de detección de vehículos se dividen en dos etapas [14]: generación de hipótesis (HG) y verificación de hipótesis (HV). En la HG, las regiones de la imagen que potencialmente contienen un vehículo son identificadas mediante un análisis rápido de la misma. En la $\mathrm{HV}$, las hipótesis son verificadas o descartadas, generalmente mediante métodos computacionalmente más intensos. La salida de la $\mathrm{HG}$ es la entrada de la $\mathrm{HV}$, por lo que su fiabilidad es importante.

Los métodos de HG se clasifican básicamente en tres categorías [14]: métodos basados en la apariencia, en estereovisión y en el movimiento. Los primeros aprovechan características físicas de los vehículos como: bordes [12], simétria [6], textura [13], luces traseras [11], sombras [9], etc. Estos métodos están condicionados por la iluminación y por los fondos de imagen saturados de objetos. Los métodos basados en estereovisión pueden explotar tanto el mapa de disparidad (DM) [2] como el mapa de perspectiva inversa (IPM) [10], ambos generados a partir de las dos imágenes (imagen izquierda y derecha). Implican la búsqueda de correspondencias entre ambas imágenes, lo cual es una tarea compleja que además conlleva una elevada carga computacional. Por último, los métodos basados en el movimiento explotan el flujo óptico generado por los vehículos en movimien- 
to, el cual se obtiene a partir del emparejamiento de píxeles en imágenes consecutivas [8]. La carga computacional es también elevada y además requieren el análisis de varias imágenes para detectar un vehículo.

En la HV, los candidatos a vehículo son confirmados o rechazados como tal. Los métodos de HV pueden estar basados en plantillas [1] o en el aprendizaje [17]. Ambos emplean la apariencia de los vehículos.

El presente artículo se centra en la etapa $\mathrm{HG}$, presentando un método de generación de hipótesis basado en la sombra que los vehículos generan bajo sí mismos. Se propone una nueva estrategia para la detección de las sombras en entornos urbanos, los cuales se caracterizan por fondos de imagen y carreteras saturados de elementos. El método propuesto esta dirigido a integrar un sistema completo de detección de vehículos (etapa HG seguida de etapa HV), el cual a su vez entrará a formar parte de un sistema anticolisión frontal.

El resto del trabajo está organizado como sigue. En la Sección 2, se presenta la sombra bajo los vehículos como característica para la detección de los mismos. La sección 3 describe el método de generación de hipótesis propuesto. Los resultados experimentales se presentan en la Sección 4 y finalmente, las conclusiones se muestran en la Sección 5.

\section{SOMBRA BAJO EL VEHÍCULO}

Un rasgo distintivo de los vehículos es la sombra que proyectan bajo sí mismos. Esta sombra se debe a que el vehículo ocluye gran parte de la luz ambiental, la cual puede estar compuesta únicamente por la luz del cielo (días nublados) o puede estar formada por la luz del cielo y la luz del sol (días soleados). Debido a la forma de los vehículos, el espacio comprendido entre la parte baja de los mismos y la superficie de la carretera es muy pequeño (menor que la altura de una rueda), por lo que tanto en condiciones nubladas como soleadas, la luz incidente bajo el vehículo es una pequeña cantidad de luz del cielo. Esto genera que la región de la carretera bajo el vehículo sea muy oscura, con poca textura y sin brillos. Incluso si el vehículo circula por una zona sombreada, la región de la carretera bajo el mismo es más oscura que sus alrededores (ver Figura 1). A partir de la sombra, la hipótesis de vehículo se genera extrayendo de la imagen la región inmediatamente superior.

El principal enfoque para la detección de la sombra bajo los vehículos se centra en la segmentación de la misma mediante umbralización por intensidad. Sin embargo, las intensidades de la sombra bajo el vehículo y de la carretera no sombreada dependen tanto del tipo de asfalto como de la iluminación, por lo que

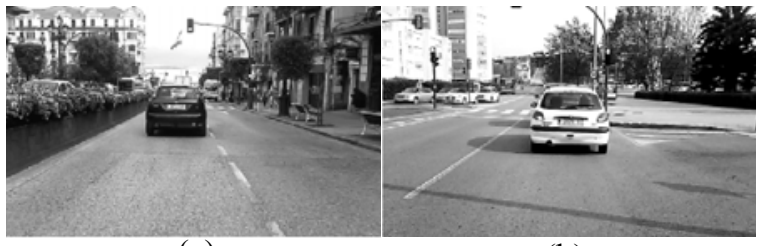

(a)

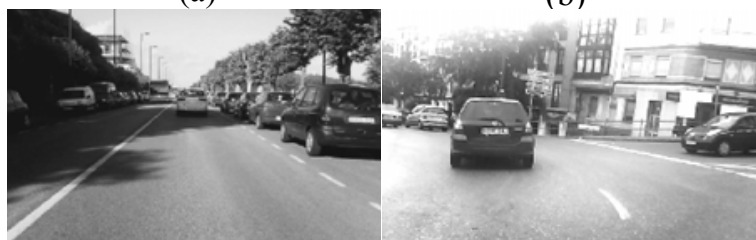

(c)
Figura 1. Sombra bajo el vehículo. (a) nublado, (b) soleado, (c) vehículo circulando en sombra y (d) lluvioso.

el umbral de intensidad que las separa no es un valor fijo. Con el fin de establecer un umbral, varios métodos se basan en el hecho de que la sombra bajo un vehículo es más oscura que la carretera no sombreada [9]. La idea es determinar un nivel de gris aproximado de la carretera, y las regiones de la imagen cuya intensidad sea menor que dicho valor tienen una elevada probabilidad de ser la sombra bajo un vehículo. En [15] se propone un método muy conocido que posteriormente es empleado en $[3,4,7,16]$, donde el nivel de gris de la carretera se obtiene a partir del espacio libre disponible de la misma frente al egovehículo. Dicho espacio es establecido como la región central de la imagen delimitada por los bordes (obtenidos por el operador Sobel). Para el nivel de gris se asume una distribución normal y la sombra bajo un vehículo queda definida como aquella región de la imagen con una intensidad menor que el umbral $m-3 \sigma$, donde $m$ y $\sigma$ son respectivamente el valor medio y la desviación típica de la intensidad del conjunto de píxeles que conforman el espacio libre de la carretera. Este método demuestra su efectividad en ciertos escenarios (autopistas y carreteras generales) y bajo determinadas condiciones atmosféricas (días nublados). Sin embargo, tanto en tráfico urbano como en condiciones soleadas, presenta ciertos problemas. Por un lado, el umbral $m-3 \sigma$ no es un límite superior de la intensidad de la sombra bajo un vehículo, sino que es un límite inferior de la intensidad de la carretera. Este hecho contribuye a falsas detecciones, ya que todos los elementos de la imagen más oscuros que la carretera (incluyendo las sombras laterales causadas por los objetos en días soleados) son clasificados como sombra bajo un vehículo. Por otro lado, la distancia entre vehículos circulando en tráfico urbano es menor que en autopistas y carreteras generales. El borde de la sombra bajo un vehículo circulando próximo al ego-vehículo, puede aparecer en la imagen como una transición gradual de intensidad cuya detección puede fallar fácilmente. Este hecho puede conducir a incluir la sombra bajo el vehículo como espacio libre de la carretera. 
El método de detección de la sombra bajo los vehículos propuesto en este artículo se basa en el método presentado por los autores en [5], en el cual se propone una estrategia de umbralización basada en la evaluación del histograma de la región de la carretera frente al ego-vehículo, donde la presencia de un vehículo delantero conlleva riesgo de colisión por alcance. Además se establece un umbral de intensidad fijo con el fin de reducir el número de falsos positivos. Este método presenta una buena tasa de detección, sin embargo, el uso de un umbral fijo limita el sistema a determinados tipos de asfalto e iluminación. Por otro lado, en condiciones soleadas los vehículos pueden proyectar una sombra lateral adyacente a sí mismos, la cual puede generar fallos en el encuadramiento de la hipótesis e incluso puede conducir a pérdidas en la detección. En el presente trabajo se abordan sendos inconvenientes mediante una estrategia basada en la aplicación de dos umbrales de intensidad. El primero se emplea con el fin de segmentar los candidatos a sombra en la región de la carretera frente al ego-vehículo y el segundo se aplica individualmente a cada candidato con el fin de separar la sombra lateral adyacente al vehículo (si la hubiere) de la sombra bajo el mismo. Finalmente, tras un filtrado morfológico en base al conocimiento previo de la pose y tamaño de las traseras de los vehículos, se generan las hipótesis definitivas. La Figura 2 ilustra los distintos pasos del método en condiciones (a) nubladas y (b) soleadas.

\section{GENERACIÓN DE HIPÓTESIS}

\subsection{REGIÓN DE INTERÉS}

Con el fin de simplificar la escena, el sistema se centra en una región de interés (ROI) correspondiente al área de la carretera frente al ego-vehículo donde la circulación de un vehículo delantero conlleva riesgo de colisión por alcance. El tráfico urbano se caracteriza por velocidades de circulación bajas $(<50 \mathrm{Km} / \mathrm{h})$, requiriendo distancias de seguridad entre vehículos menores que en carreteras nacionales y autopistas. Así, la ROI es considerada una región de la carretera de $15 \mathrm{~m}$ de longitud y una anchura igual a la del egovehículo. Debido a la perspectiva y asumiendo condición de tierra plana, la ROI en la imagen es una región trapezoidal que cubre la zona de la carretera frente al ego-vehículo (Figura 2(a)).

\subsection{DETECCIÓN DE LA SOMBRA}

La detección de la sombra del vehículo delantero circulando dentro de la ROI se realiza mediante umbralización por intensidad, en base a que la sombra bajo el mismo es generalmente uno de los elementos más oscuros de la ROI. Además de la sombra y del asfalto no sombreado, en la ROI pueden estar presen-

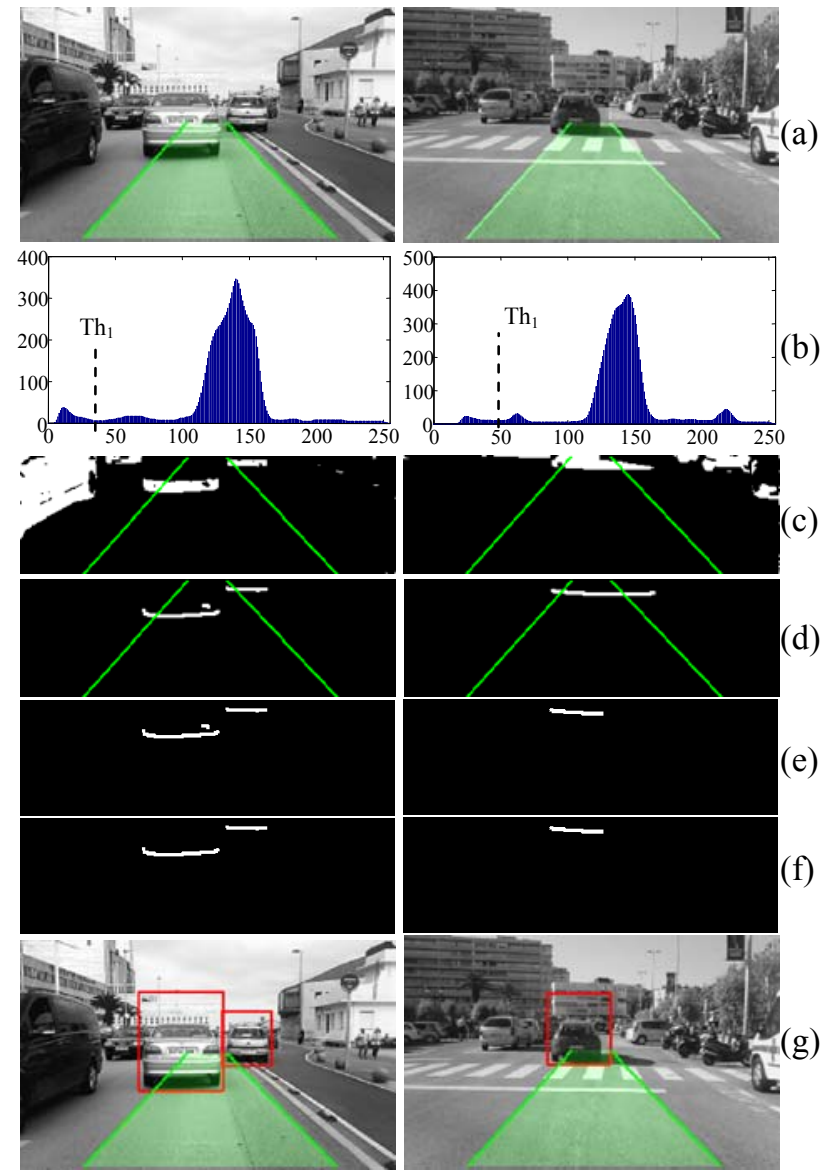

Figura 2. Escena nublada (izquierda) y soleada (derecha). (a) imagen inicial y ROI, (b) histograma de la ROI, (c) $M(x, y)$ tras la umbralización con $T h_{l}$, (d) bordes horizontales dentro de la ROI, (e) $M(x, y)$ tras la umbralización con $T h_{2}$, (f) $M(x, y)$ tras el filtrado morfológico y $(\mathrm{g})$ hipótesis generadas.

tes otros elementos como sombras laterales causadas por los objetos de la escena (edificios, árboles, etc), indicaciones pintadas (líneas de carríl, pasos de cebra, etc), bordillos, etc. Sin embargo, se observa que:

1) La carretera, las sombras laterales y la sombra bajo un vehículo son la misma superficie (asfalto) pero con distinta iluminación. La cantidad de luz ambiental incidente en la región de la carretera bajo un vehículo es inferior a la incidente sobre la sombra lateral causada por un objeto, la cual es a su vez inferior a la incidente en la carretera no sombreada. Por lo tanto, la intensidad de un píxel de la imagen perteneciente a la sombra bajo un vehículo es menor que la de los píxeles correspondientes a una sombra lateral y a la carretera no sombreada.

2) La intensidad de un píxel de la sombra bajo un vehículo es menor que la de un píxel perteneciente a: indicaciones pintadas en la carretera (blancas, amarillas y azules), manchas en el asfalto iluminadas por la luz ambiental [5,9] y bordillos (la cara vertical del 
bordillo está sombreada debido a la oclusión de una mitad del hemisferio del cielo).

De este modo, cuando un vehículo delantero circula dentro de la ROI, el histograma del conjunto de píxeles que componen la misma presenta dos picos de intensidad característicos (ver Figura 2(b)). El pico con el menor nivel de gris corresponde a los píxeles que conforman la sombra bajo el vehículo, mientras que el pico con mayor nivel de gris se debe a los píxeles que componen la carretera no sombreada. Los niveles de gris correspondientes a brillos e indicaciones pintadas en el asfalto son mayores que los de la propia carretera no sombreada, por lo que en el histograma se localizan (si los hubiere) a la derecha del pico debido a esta última (ver Figura 2(b) derecha). En cambio, los píxeles pertenecientes a sombras laterales y manchas en el asfalto (si los hubiere) se localizan entre ambos picos característicos. Dependiendo de la iluminación, los niveles de gris de los distintos elementos de la ROI se desplazan a lo largo del histograma pero la distribución de éste no varía. Por lo tanto, la estrategia para la segmentación de la sombra bajo los vehículos consiste en considerar en primer lugar, el máximo local $P$ con menor valor de intensidad del histograma $h(I)$, es decir, el pico más a la izquierda.

$$
\begin{aligned}
& \text { for } I=3: 253 \\
& \quad \text { if } h(I)>h(I-1) \text { and } h(I-1)>h(I-2) \text { and } \\
& \quad h(I)>h(I+1) \text { and } h(I+1)>h(I+2) \\
& \quad P=I ; \text { break; } \\
& \quad \text { end }
\end{aligned}
$$

A partir de $P$, el umbral $T h_{1}$ es establecido como el valor del nivel de gris correspondiente al mínimo local inmediatamente a su derecha (ver Figura 2(b)),

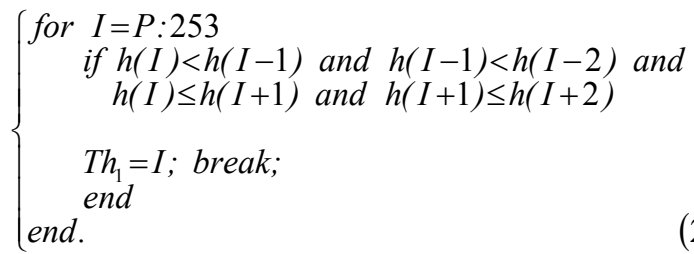

Así, las regiones de la carretera cuyo nivel de gris sea inferior a $T h_{l}$ son candidatas a ser sombra bajo un vehículo, mientras que todas aquellas cuya intensidad sea superior son descartadas. En el caso en que el vehículo delantero circule parcialmente dentro de la región de interés, su sombra no aparecerá completa dentro de la ROI (ver Figura 2(a) izquierda). Con el fin de segmentar la sombra completa, la umbralización se lleva a cabo en la región de la imagen que abarca la longitud de la ROI y una anchura igual a la anchura de la imagen adquirida. El resultado es una máscara binaria $M(x, y)$ con las regiones candidatas a ser sombra bajo un vehículo (ver Figura 2(c)). Para obtener las hipótesis se consideran los bordes horizontales inferiores de cada región resultante, los cuales corresponden a la transición vertical entre la sombra bajo el vehículo (arriba) y la carretera no sombreada (abajo). Los vehículos circulando bien total o parcialmente dentro de la ROI son susceptibles de una colisión por alcance, por lo que únicamente los bordes horizontales total o parcialmente dentro de la ROI son candidatos (ver Figura 2(d)).

En condiciones nubladas la umbralización conduce generalmente a una correcta segmentación de la sombra bajo los vehículos (ver Figura 2 izquierda). Sin embargo, en días soleados el vehículo puede proyectar una sombra lateral adyacente a la sombra bajo el mismo (ver Figuras 2 derecha y 3 ). La intensidad de la sombra lateral es superior a la de la sombra bajo el vehículo ya que la cantidad de luz ambiental iluminando la primera es mayor que la incidente sobre la segunda. Sin embargo, aún siendo mayor, puede alcanzar valores cercanos a los de la sombra bajo el mismo, pudiendo satisfacer $T h_{l}$ y siendo incluída como sombra bajo el vehículo (ver Figura 2(d) derecha). Este hecho puede conducir a un fallo en el encuadramiento de la hipótesis e incluso a una pérdida en la detección.

Con el fin de identificar la sombra adyacente, se propone un segundo umbral de intensidad para cada borde horizontal presente en la ROI. En un borde horizontal generado por la sombra bajo un vehículo y por una sombra lateral adyacente, las intensidades de los píxels superiores del borde correspondientes a la sombra bajo el vehículo tienen un valor similar, el cual además es menor que la intensidad de los píxeles superiores del borde correspondiente a la sombra

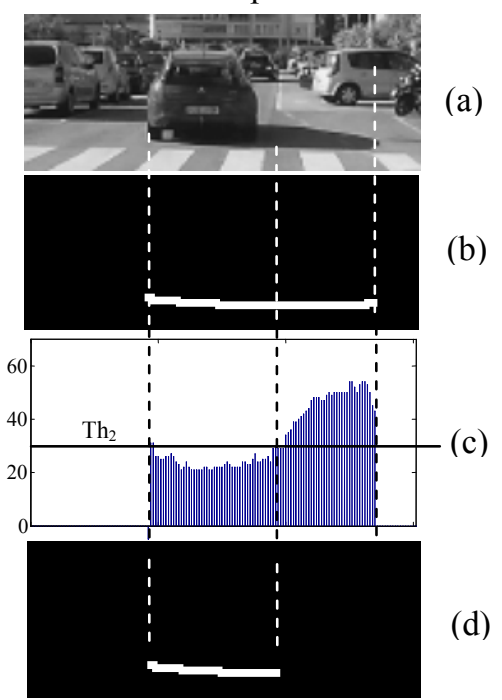

Figura 3. Umbralización del borde horizontal de la Figura 2 derecha: (a) imagen inicial, (b) borde horizontal tras la umbralización con $T h_{l}$, (c) intensidad de los píxeles superiores del borde y (c) borde resultante tras la umbralización con $T h_{2}$. 
lateral (ver Figura 3(c)). De este modo, el valor medio de intensidad del conjunto compuesto por los píxeles superiores del borde es un límite superior para la sombra bajo los vehículos, y por lo tanto es un umbral $T h_{2}$ que las separa,

$$
T h_{2}=\frac{1}{n_{p}} \cdot \sum_{i=1}^{n_{p}} I_{i}\left(x_{s}, y\right),
$$

donde $n_{p}$ es el número total de píxeles superiores del borde, $x$ e $y$ son la fila y columna respecto a la esquina arriba-izquierda, e $I_{i}\left(x_{s}, y\right)$ es la intensidad del píxel superior del píxel-borde bajo evaluación.

Para determinar si el borde horizontal se debe únicamente a la sombra bajo el vehículo o si por el contrario también es debido a una sombra lateral adyacente, se evalúa la desviación típica $\sigma$ del conjunto de píxeles superiores del borde, la cual indica la dispersión de los valores del conjunto respecto al valor medio,

$$
\sigma=\sqrt{\frac{1}{n_{p}-1} \cdot \sum_{i=1}^{n_{p}}\left(I_{i}\left(x_{s}, y\right)-T h_{2}\right)^{2}}
$$

Un valor bajo de $\sigma$ indica que el valor de las intensidades de los píxeles superiores es similar (borde debido únicamente a la sombra bajo el vehículo), mientras un valor alto indica que las intensidades de los píxeles superiores del borde difieren notablemente las unas de las otras (borde horizontal debido en parte a la sombra bajo el vehículo y en parte a la sombra lateral adyacente). En base a resultados empíricos, se considera el primer caso y por tanto no se aplica $T h_{2}$ si $\sigma$ es menor que el $30 \%$ del valor medio de intensidad, $\sigma<0.3 \cdot T h_{2}$. Por el contrario, si $\sigma \geq 0.3 \cdot T h_{2}$ estamos ante el segundo caso y por tanto si se aplica el umbral $T h_{2}$,

$$
M(x, y)= \begin{cases}1, & \sigma<{ }^{T h_{2}} / 3 \\ & o\left(\sigma \geq^{T h_{2}} / 3 \text { y } \quad I_{i}\left(x_{S}, y\right)<T h_{2}\right) \\ 0, & \text { en otro caso. }\end{cases}
$$

Las Figuras 2(e) derecha y 3(d) muestran la máscara binaria $M(x, y)$ resultante tras la umbralización con $\mathrm{Th}_{2}$. El borde horizontal en la Figura 2(d) izquierda es debido únicamente a la sombra bajo el vehículo, satisfaciendo $\sigma<0.3 \bullet T h_{2}$ por lo que no se le aplica el umbral $T h_{2}$ (ver Figura 2(e) izquierda).

\subsection{FILTRADO MORFOLÓGICO Y GENERACIÓN DE HIPÓTESIS}

Tras la umbralización por intensidad, las hipótesis a vehículo finales se obtienen mediante un filtrado morfológico en base al conocimiento de la anchura del vehículo en la imagen. Debido a la perspectiva, la anchura de un vehículo delantero varía respecto a su posición vertical en la imagen. La anchura de los vehículos depende de la marca y modelo por lo que una anchura estandar $v_{\text {width }}$ es considerada igual a la anchura de la ROI. Por lo tanto, para cada posición vertical en la imagen, la $v_{\text {width }}$ viene directamente definida por la anchura de la ROI en esa misma posición vertical. Así, los bordes horizontales en $M(x, y)$ cuya anchura se encuentren entre el $120 \%$ y el $80 \%$ de $v_{\text {width }}$ en la posición vertical computada, son considerados candidatos finales a ser bordes debidos a la sombra bajo un vehículo (ver Figura 2(f)).

Finalmente, la hipótesis se genera extrayendo de la imagen la región rectangular inmediatamente superior al borde horizontal. Con el fin de encuadrar correctamente la trasera del vehículo, la anchura del borde es ampliada un 5\% tanto a la izquierda como a la derecha y una relación de aspecto estandar para las traseras de los vehículos es asumida. Se considera que la altura del marco es igual al $130 \%$ de la anchura. Esta relación de aspecto asegura el correcto encuadramiento de vehículos altos (furgonetas). La Figura 2(g) muestra las hipótesis de vehículo finales.

\section{RESULTADOS}

Los ensayos se realizaron en tráfico urbano bajo condiciones nubladas y soleadas en Santander. La cámara empleada es una Trust Widescreen HD WebCam, 8 bit píxel, autobalance de blancos y tiempo de exposición automático, la cual proporcionó imágenes en escala de grises de $240 \times 320$. Un total de 13,200 imágenes fueron adquiridas y procesadas en Matlab ${ }^{\circledR}$ sobre un ordenador portátil convencional.

La Figura 4 muestra algunos ejemplos de los resultados obtenidos en la generación de hipótesis. El método demuestra gran robustez, ya que detecta correctamente las sombras bajo los vehículos en las diversas condiciones de iluminación. Además, la estrategia de umbralización propuesta hace el método robusto ante sombras laterales e indicaciones en el asfalto, minimizando el número de pérdidas en la detección. Por otro lado, se puede apreciar que los vehículos delanteros tanto alineados como desalineados respecto al ego-vehículo, son correctamente enmarcados.

Los resultados cuantitativos son mostrados en la Tabla 1, donde las tasas de detección de positivos $P R$ y de falsos positivos FPR son definidas como

$$
P R(\%)=\frac{P}{V} \cdot 100, \quad F P R(\%)=\frac{F P}{H} \cdot 100,
$$

donde $V$ es el número total de vehículos dentro de la ROI y $H$ es el número total de hipótesis generadas. 


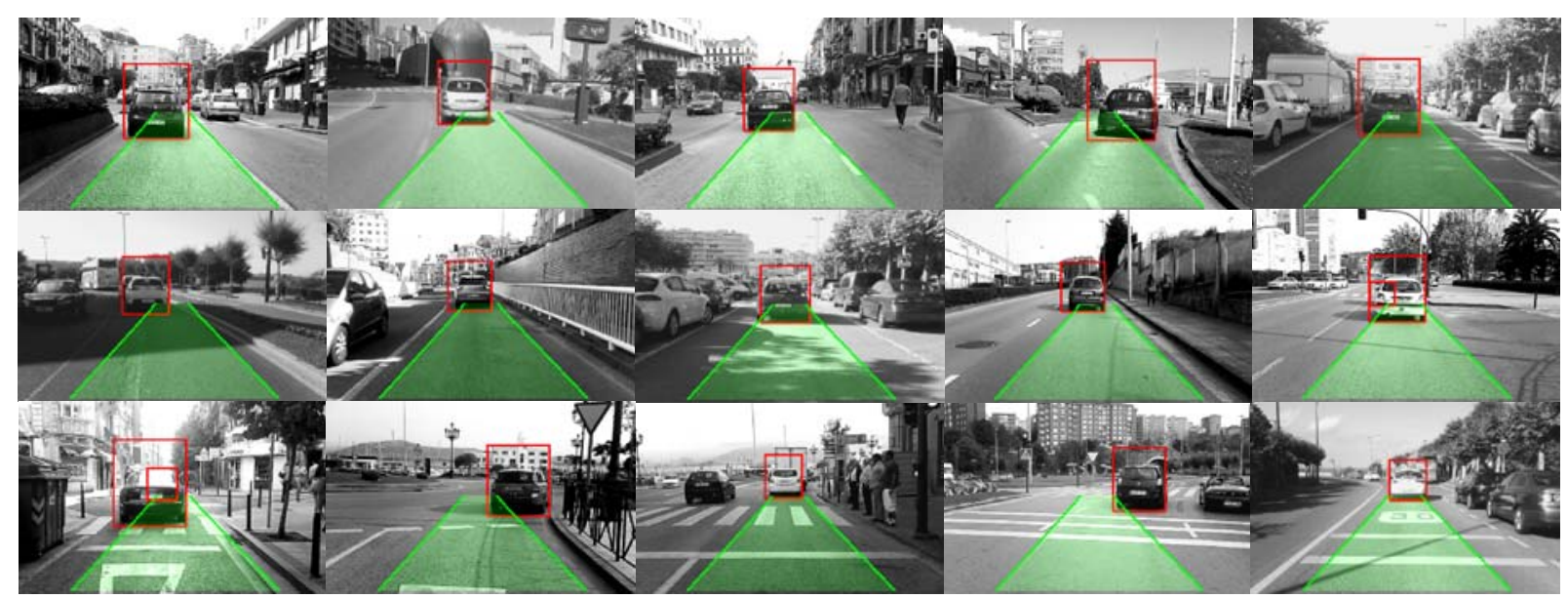

Figura 4. Ejemplos de los resultados de generación de hipótesis obtenidos en días nublados y soleados, con el vehículo delantero desalineado y en presencia de sombras laterales e indicaciones pintadas en el asfalto .

Tabla 1: Resultados de la generación de hipótesis.

\begin{tabular}{|l|c|c|}
\hline & nublado & soleado \\
\hline Número total de imágenes & 7,920 & 5,806 \\
Número total de vehículos en la ROI (V) & 7,303 & 5,115 \\
Número total de hipótesis generadas (H) & 7,590 & 5,452 \\
Positivos (P) & 7,153 & 4,992 \\
Falsos positivos (FP) & 437 & 470 \\
Falsos negativos (FNVM) & 150 & 123 \\
Tasa de positivos (PR) & $97.94 \%$ & $97.59 \%$ \\
Tasa de falsos positivos (FPR) & $5.75 \%$ & $8.6 \%$ \\
\hline
\end{tabular}

Los resultados muestran una elevada tasa de detección de positivos $P R$, alcanzando el $97.94 \%$ y el $97.59 \%$ en condiciones nubladas y soleadas. La pérdida en la detección no se debe principalmente a vehículos no detectados sino a vehículos incorrectamente encuadrados. En ambas condiciones atmosféricas, el número de vehículos no detectados es pequeño lo que demuestra que el método de generación de hipótesis es muy fiable. En cuanto a los falsos positivos, el número de hipótesis conteniendo a un no-vehículo FP es relativamente bajo para una fase previa de la detección como es la HG, alcanzando unas tasas de FPR del $5.75 \%$ y del $8.6 \%$ para condiciones nubladas y soleadas, respectivamente. El filtro morfológico y la consideración de la ROI contribuye en gran medida a la baja tasa de falsos positivos.

El método propuesto es comparado con el presentado en [15] y descrito en la Sección 2, donde tras el umbralizado, se emplea un filtro morfológico y se eliminan los bordes generados por sombras laterales. Desafortunadamente, estas dos últimas etapas no son proporcionadas en [15], por lo que una comparación cuantitativa entre ambos métodos no es posible. Nos centramos así en comparar únicamente el umbral de intensidad $T h_{15}=m-3 \sigma$ propuesto en [15] y el umbral $T h_{1}$ propuesto en el presente trabajo, mediante dos ejemplos ilustrativos.

La Figura 5(a) muestra una imagen adquirida en un día nublado. El metodo propuesto en [15] establece el

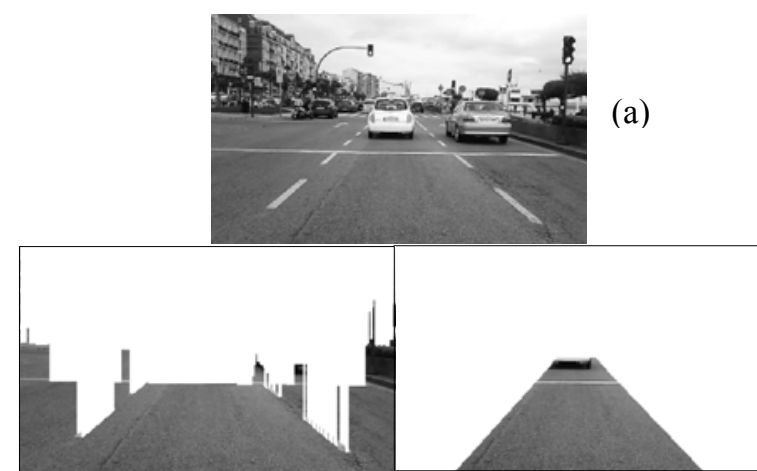

(b)

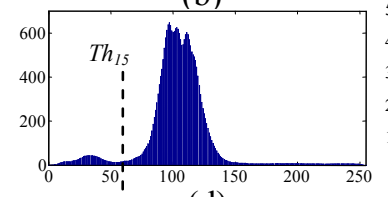

(d)

(c)

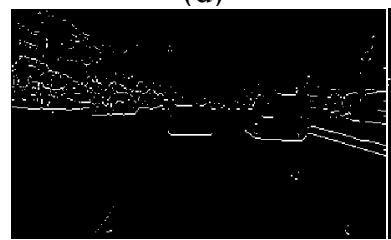

(f)

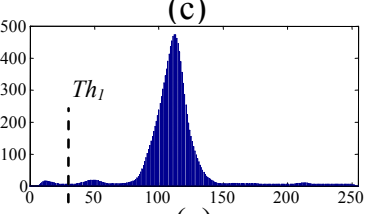

(e)

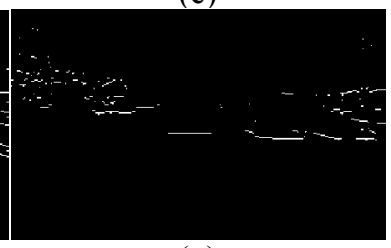

(g)

Figura 5. (a) imagen adquirida. Izquierda, método de [15]: (b) espacio libre disponible, (d) su histograma, (f) mapa de bordes tras la umbralización con $T h_{15}$. Derecha, método propuesto: (c) ROI, (e) su histograma y (g) mapa de bordes obtenidos tras la umbralización con $T h_{l}$.

umbral de intensidad que separa la carretera y la sombra bajo los vehículos $T h_{15}$ como el límite inferior de la intensidad del espacio libre de la carretera (ver Figura 5(b)), proporcionando unos valores $m_{15}=105.87, \sigma_{15}=15.27$ y $T h_{15}=60.04$ (ver Figura $5(\mathrm{~d})$ ). Los bordes cuya región superior presentan una intensidad menor que el umbral $T h_{15}$ son clasificados como candidatos a ser la sombra bajo un vehículo (ver la Figura 5(f)). Por otro lado, el umbral de inten- 
sidad que se propone en este trabajo, es establecido considerando únicamente el conjunto de píxels de la ROI (ver la Figura 5(c)). Obtenemos un valor umbral $T h_{l}=28$, (ver la Figura 5(e)). De este modo, todas aquellas regiones de la imagen con una intensidad menor que el umbral $T h_{l}$ son clasificadas como sombra bajo un vehículo. La Figura 5(g)) muestra el mapa de bordes inferiores extraídos de las regiones candidatas. Si comparamos ambos umbrales (ver la Figuras 5(d) y (e)), el umbral $T h_{l}$ es más cercano al nivel de intensidad de las sombras bajo los vehículos que el umbral $T h_{15}$, y por lo tanto, la umbralización realizada con $T h_{1}$ es más efectiva, descartando un mayor número de bordes que no son debidos a la sombra bajo un vehículo (ver Figuras 5(f) y (g)). Este hecho pasa a tener mucha más relevancia en escenas con presencia de sombras laterales o manchas oscuras en el asfalto.

La Figura 6(a) muestra una imagen adquirida en un día soleado en presencia de sombras laterales causadas por los vehículos aparcados. De acuerdo con el método propuesto en [15], a partir de los píxels que conforman el espacio libre de conducción frente al ego-vehículo, obtenemos $m_{15}=170.87, \sigma_{15}=15.43 \mathrm{y}$ $T h_{15}=124.67$. Como se puede observar en la Figura 6(d), el límite inferior de intensidad de los píxeles de la carretera no sombreada es mayor que el nivel de intensidad de las sombras laterales, por lo que los bordes de las mismas son clasificados como aquellos debidos a la sombra bajo un vehículo (ver Figura 6(f)). Por otro lado, el método propuesto proporciona un umbral $T h_{l}=45.61$ (ver Figura 6(e)). El umbral $T h_{l}$ es considerablemente menor que el umbral $T h_{15} \mathrm{y}$ menor que el nivel de intensidad de la mayor parte de las sombras laterales, descartándolas como posibles candidatos (ver Figura 6(g)). El diferente fucionamiento de $T h_{15}$ y $T h_{1}$ puede ser apreciado en las Figuras $6(f)$ y $6(\mathrm{~g})$.

Mediante un amplio rango de imágenes de test, se verificó que el umbral de intensidad propuesto para segmentar la sombra bajo los vehículos es más preciso que el presentado en [15], proporcionando mejores resultados, especialmente en días soleados en presencia de sombras laterales.

\section{CONCLUSIONES}

Se ha presentado un método de generación de hipótesis para la detección de vehículos delanteros con el fin de evitar colisiones por alcance. Las hipótesis son generadas en base a la sombra que los vehículos proyectan bajo sí mismos. El método supera importantes dificultades como la presencia de sombras laterales, indicaciones pintadas en la carretera y fondos de imagen saturados de objetos. Los ensayos llevados a cabo en tráfico urbano bajo diferentes

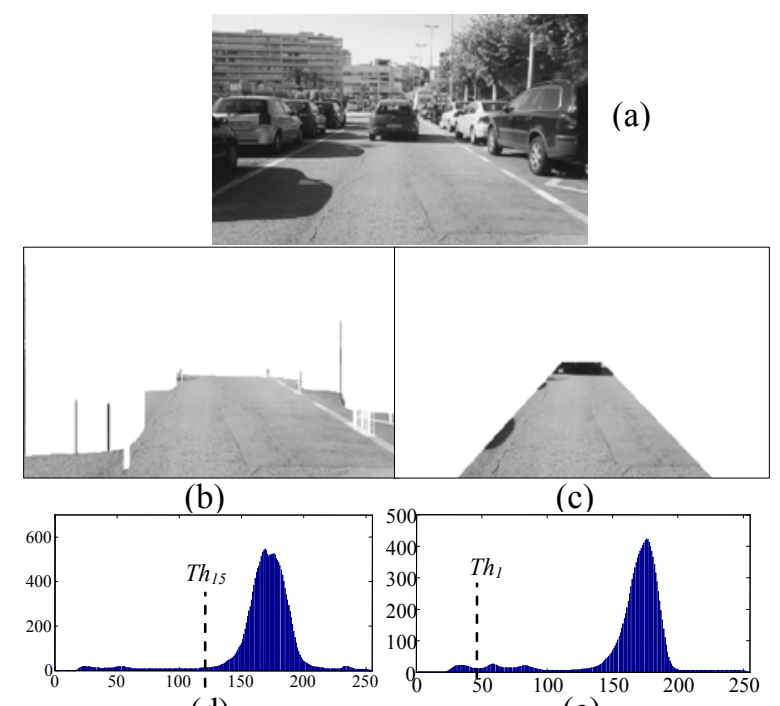

(d)

(e)

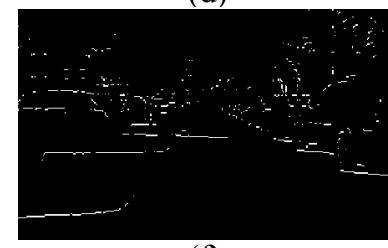

(f)

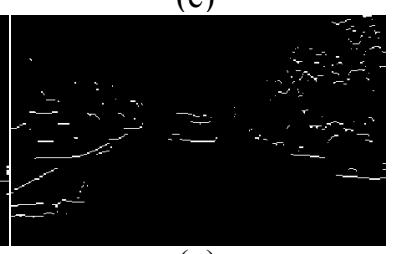

(g)
Figura 6. (a) imagen adquirida. Izquierda, método de [15]: (b) espacio libre disponible, (d) su histograma, (f) mapa de bordes tras la umbralización con $T h_{15}$. Derecha, método propuesto: (c) ROI, (e) su histograma y (g) mapa de bordes obtenidos tras la umbralización con $T h_{l}$.

condiciones ambientales, demuestran una notable robustez y efectividad del método propuesto, proporcionando una elevada tasa de detección tanto en condiones nubladas como soleadas. El número de falsos positivos es relativamente bajo para una fase previa de la detección como es la HG. El filtro morfológico y la consideración de la ROI contribuye en gran medida a esta baja tasa de falsos positivos.

Por otro lado, la estrategia de umbralización propuesta alcanza mejores resultados que el método propuesto en [15], rechazando un mayor número de bordes que no son debidos a la sombra bajo un vehículo, especialmente en días soleados con presencia de sombras laterales en la carretera.

Como trabajo futuro, nos centraremos en la etapa de verificación de hipótesis, con el fin de desarrollar un sistema completo de detección de vehículos delanteros en la trayectoria.

\section{Agradecimientos}

Este trabajo ha sido realizado bajo el patrocinio del Ministerio de Economía y Competitividad. (Proyecto: DPI2012-36959). 


\section{Referencias}

[1] Bensrhair, A., Bertozzi, M., Broggi, A., et al, (2001) "A Cooperative Approach to VisionBased Vehicle Detection", in: Proc. IEEE Intell. Transport. Syst. Conf., pp. 207-212.

[2] Bertozzi, M., Broggi, A., (1998) "GOLD: A Parallel Real-Time Stereo Vision System for Generic Obstacle and Lane Detection", IEEE Trans. Image Process., pp. 62-81.

[3] Handman, U., Kalinke, T., Tzomakas, C., Werner, Mn, Seelen, W.v., (2000) "An Image Processing System for Driver Assistance", Image and Vision Computing, pp. 367-376.

[4] Hoffmann, C., Dang, T., Stiller, C., (2004) "Vehicle detection fusing 2D visual features", in: Proc. IEEE Intelligent Vehicles Symposium, pp. 280-285.

[5] Ibarra, M., Pérez, J.M., Torre, C., Alonso, L., (2014) "MonoVision-Based Vehicle Detection, Distance and Relative Speed Measurement", in Urban Traffic, IET Intell. Trans. Syst., pp. 655664.

[6] Kuehnle, A., (1991) "Symmetry-based recognition for vehicle rears", Pattern Recognition Letters, pp. 249-258.

[7] Lim, K.H., Ang, L.M., Seng, K.P, Chin, S.V., (2009) "Lane-vehicle detection and tracking", in: Proc. Int. Conf. of Engineers and Comp. Scientist, vol. II.

[8] Michalke, T., Stein, F., Franke, U., (2011) "Towards a closer fusion of active and passive safety: Optical flow-based detection of vehicle side collisions", in: Proc. Intell. Vehicles Symp., pp. 181-188.

[9] Mori, H., Charkai, N., (1993) "Shadow and Rhythm as Sign Patterns of Obstacle Detection", in: Proc. Int. Symp. Ind. Electronics, pp. 271-277.

[10] Nedevschi, S., Danescu, R., Marita, T., et al, (2007) "A Sensor for Urban Driving Assistance Systems Based on Dense Stereovision", in: IEEE Intell. Vehicles Symp., pp. 276-283.

[11] O’Malley, R., Jones, E., Glavin, M., (2010) "Rear-Lamp Vehicle Detection and Tracking in Low-Exposure Color Video for Night Conditions", IEEE Trans. Intell. Transport. Syst., pp. 453-462.
[12] Parodi, P., Piccioli, G., (1995) "A feature-based recognition scheme for traffic scenes", in: Proc. Intell. Veh. Symp. Detroit, pp. 229-234.

[13] Peiqun, L., Jianmin, X., Jianyong, B., (2007) "Robust Vehicle Detection in Vision Systems Based on Fast Wavelet Transform and Texture Analysis", in: IEEE Int. Conf. on Automation and Logistics, pp. 2958-2963.

[14] Sun, Z., Bebis, G., Miller, R., (2006) "On-road vehicle detection: a review", IEEE Trans. on Pattern Anal. and Mach. Intell., pp. 694-711.

[15] Tzomakas, C., Seelen, W., (1998) "Vehicle Detection in Traffic Scenes using Shadows", Tech. Rep. 98-06, Institut FurNeuroinformatik, Ruht-universitat, Bochum, Germany.

[16] Van Leeuwen, M.B., Groen, F.C.A., (2005) "Vehicle Detection with a Mobile Camera, Spotting Midrange, Distant and Passing Cars", IEEE Robotics and Automation Magazine, pp. 37-43.

[17] Zhang, Z., Tan, T., Huang, K., Wang, Y., (2012) "Three-dimensional deformable-modelbased localization and recognition of road vehicles", IEEE Trans. on Image Process., pp. 1-13. 